\title{
交变负载下变齿厚齿轮副侧隙控制试验研究*
}

\author{
邹帅东 1,2 王光建 ${ }^{1,3}$ 蒋宇将 ${ }^{1,2}$ 任品旭 ${ }^{4}$ 喻 立 $^{1,2}$ 李小兵 ${ }^{5}$ \\ (1. 重庆大学机械传动国家重点实验室 重庆 400044; \\ 2. 重庆大学机械工程学院 重庆 400044; \\ 3. 重庆大学汽车工程学院 重庆 400044;
}

4. 卡内基梅隆大学梅隆科学院 匹兹堡 15217 美国;

5. 工业和信息化部电子第五研究所 广州 510000)

\begin{abstract}
摘要: 提出一种基于变齿厚齿轮的齿轮侧隙控制方法: 在取得变齿厚齿轮副连续侧隙曲线规律基础上, 根据主动轮的角位移 来实时调整变齿厚齿轮主动轮与从动轮的相对位置关系进行侧隙控制; 推导了具有偏心误差齿轮副的双向无负载传动误差及 侧隙计算公式及其等效关系, 在此基础上根据双向传动误差试验曲线获得了齿轮副连续侧隙曲线; 在未消隙情况下, 分析了 不同负载幅值及不同负载频率的传动误差试验曲线，结果表明在交变负载下侧隙对齿轮副的动态传动误差有明显影响; 在不 同负载条件下, 开展了基于变齿厚齿轮副的侧隙电控试验研究, 对比其动态传动误差试验曲线, 结果表明: 电控侧隙控制后, 因侧隙和脱啮导致的动态传动误差跳变或波动现象明显改善, 动态传动误差明显减小，证明该侧隙控制方法有效。
\end{abstract}

关键词：侧隙控制；连续侧隙；双向传动误差；交变负载；单/双边冲击

中图分类号: $\mathrm{TH} 13$

\section{Experimental Investigation on Backlash Control of Variable Tooththickness Gear Pair under Varying Load Excitation}

\author{
ZOU Shuaidong $^{1,2}$ WANG Guangjian ${ }^{1,3}$ JIANG Yujiang ${ }^{1,2} \quad$ REN Pinxu $^{4}$

$$
\mathrm{YU} \mathrm{Li}^{1,2} \text { LI Xiaobing }{ }^{5}
$$

(1. State Key Laboratory of Mechanical Transmissions, Chongqing University, Chongqing 400044;

2. College of Mechanical Engineering, Chongqing University, Chongqing 400044;

3. School of Automotive Engineering, Chongqing University, Chongqing 400044;

4. Mellon College of Science, Carnegie Mellon University, Pittsburgh 15217, USA;

5. The Fifth Electronic Institute, Ministry of Industry and Information Technology, Guangzhou 510000)

\begin{abstract}
A backlash control method based on the variable tooth thickness gear pair (VTTGP) is presented. On the basis of the continuous backlash curve of VTTGP, the backlash compensation is carried out in real time according to the angular displacement of the driving gear by adjusting the relative position of driving and driven gear. The two-directions transmission error, backlash and their relationship of an involute gear pair with eccentricities are developed. On this basis, the continuous backlash curve of gear pair is obtained according to the experimental two-directions transmission error curve. The dynamic transmission error (DTE) curves of VTTGP under different loads with various amplitude and frequency are obtained by experimental method, and the results show that the backlash has a significant influence on the gear pair's DTE. An experimental investigation on electrical control to backlash based on VTTGP is carried out under different varying load and the DTE is analyzed. The results show that the jump or fluctuation phenomenon of DTE is obviously improved after backlash control, which validates the electrical anti-backlash method.
\end{abstract}

Key words: backlash control; continuous backlash; two-directions transmission error; varying load; one/double sided gear rattle

* 国家自然科学基金(51275538)、上海市空间飞行器机构重点实验室开放 课题(SM2014D101)和 2016 年工业转型升级(中国制造 2025)重点 (0747-1660SITCA120/包件 2)资助项目。20190910 收到初稿, 20191220 收到修改稿 


\section{0 前言}

对于精密伺服传动系统, 传动误差和侧隙是影 响运动准确性的重要因素。侧隙直接影响伺服控制 的稳定性、精度和系统敏感度。在变负载条件下, 齿轮侧隙易导致运动不连续, 冲击等现象 ${ }^{[1-2]}$ 。因此, 调整和控制齿轮侧隙具有重要意义。在实际应用中, 除提高加工和装配精度外, 还需要采取其他的消隙 机构和技术措施消除侧隙 ${ }^{[3]}$ 。常用的消隙方法有: 中心距调整法、双片齿轮弹簧消隙法等, 但这些方 法存在只能消除常值侧隙或效率低等问题 ${ }^{[4]}$ 。随着 控制技术的发展，通过控制算法或通过机械与控制 结合的消隙方式目前是研究热点之一。文献[5]提出 柔度概念, 运用反推控制线性化处理齿轮传动伺服 系统的侧隙非线性, 实现齿轮侧隙非线性补偿。文 献[6]针对低速往复运动系统, 通过输入速度的开环 修正进行侧隙补偿, 但该消隙方法对电动机的响应 性要求非常高, 抗干扰能力低。文献[7]提出基于压 电陶瓷的超精密丝杜消隙机构, 能根据实际工况控 制压电陶瓷的伸缩以消除侧隙, 但限于轻载条件下 的应用。目前在数控机床、跟踪系统中应用的双电 动机驱动电消隙方式采用偏置力矩或转速差进行消 隙控制 ${ }^{[8-9]}$, 但由于使用两套传动链、两套伺服系统 以及消隙控制卡，体积质量大、成本较高。

在精密齿轮传动系统中, 偏心是大周期的时变 传动误差和周期性时变侧隙的主要来源 ${ }^{[1]}$, 若能找 到包括常值侧隙和周期性时变侧隙在内的总侧隙规 律曲线, 则根据变齿厚齿轮轴向相对移动可调整侧 隙的特点可进行侧隙调整和控制。为此, 本文提出 一种基于变齿厚齿轮传动的电控消隙方法: 在取得 变齿厚齿轮副连续侧隙曲线规律基础上, 根据主动 轮的角位移来实时调整变齿厚齿轮主动轮与从动轮 的相对位置关系进行侧隙控制。在推导具有双偏心 误差齿轮副的理论无负载传动误差及侧隙计算公式 及其等效关系基础上, 则可通过测试双向传动误差 得到齿轮副连续时变侧隙曲线。为此, 根据变齿厚 主动轮实时角位移, 则可确定齿轮副实时侧隙, 从 而调整和控制变齿厚齿轮的相对轴向位置即可实现 齿轮副侧隙控制和调整。同时, 在交变负载条件下, 开展了相应的侧隙控制测试。

\section{1 变齿厚齿轮副侧隙控制原理及策略}

变齿厚齿轮副的侧隙控制原理是基于变齿厚齿 轮齿厚沿轴向方向变化的特点, 通过调整主动轮和
从动轮之间的相对轴向位置控制啮合侧隙大小，如 图 1 所示。本文设计的变齿厚齿轮为复合变位变齿 厚齿轮 ${ }^{[10]}$, 且左右齿面切向变位线性变化且变化斜 率相同。因此, 轴向位移与侧隙的关系为

$$
j=2 s \tan \beta
$$

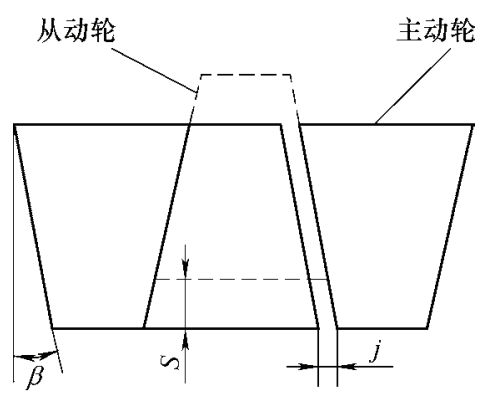

图 1 复合变位变齿厚齿轮副消隙原理

式中, $j$ 为齿轮副侧隙值; $s$ 为消隙所需轴向位移; $\beta$ 为新型变齿厚齿轮左右螺旋角。因此。在取得变 齿厚齿轮副连续侧隙曲线后，在确定主从动轮的啮 合相位的前提下，则在每一个啮合位置可以知道其 实际侧隙值, 通过式(1)调整变齿厚齿轮的相对位置 即可控制啮合侧隙。

图 2 所示为基于主动轮角位移判断的调隙流程

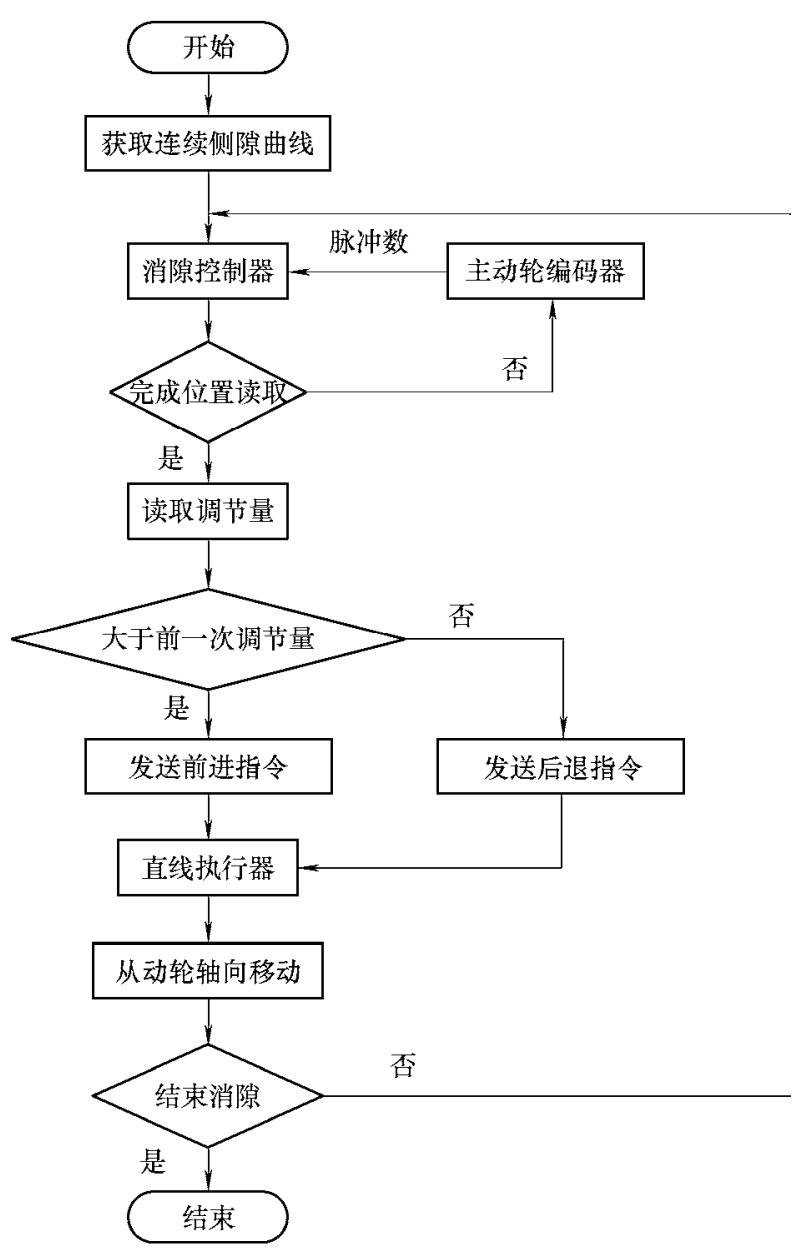

图 2 基于主动轮角位移控制的消隙流程图 
图, 在取得连续侧隙曲线后, 在齿轮传动过程中, 根据主动轮编码器的实时角位移/位置值可知实时 侧隙值, 则可实时地控制调整主从动齿轮的相对位 置进行侧隙控制。

\section{2 双向无负载传动误差与侧隙等效 关系}

\section{1 双向无负载传动误差理论计算}

定义因轴承、轴及齿轮等加工及装配误差导致 主动轮几何中心与其实际旋转中心的偏移量 $e_{1}$ (图 3) 为主动轮综合偏心误差, 啮合线增量计算如下 ${ }^{[1,11]}$

$$
\left[\begin{array}{c}
\Delta F_{1 n} \\
\Delta F_{1 s} \\
\Delta F_{1 n}^{0} \\
\Delta F_{1 s}^{0}
\end{array}\right]=e_{1}\left[\begin{array}{c}
\sin \left(\varphi_{1 c c}+\theta_{1}+\alpha\right) \\
-\sin \left(\varphi_{1 c}-\theta_{1}+\alpha\right) \\
\sin \left(\theta_{1}+\alpha\right) \\
-\sin \left(-\theta_{1}+\alpha\right)
\end{array}\right]
$$

式中, $\Delta F_{1 n}$ 为主动轮逆时针旋转时偏心导致的啮合 线增量; $\Delta F_{1 s}$ 为主动轮顺时针旋转时偏心导致的啮 合线增量; $\Delta F_{1 n}^{0}$ 为主动轮逆时针旋转初始时刻时偏 心导致的啮合线增量; $\Delta F_{1 s}^{0}$ 为主动轮顺时针旋转初 始时刻时偏心导致的啮合线增量; $\varphi_{1 c c}$ 为主动轮逆 时针转动任意时刻的转动角位移; $\varphi_{1 c}$ 为主动轮顺时 针转动任意时刻的转动角位移; $e_{1}$ 和 $\theta_{1}$ 为主动轮偏

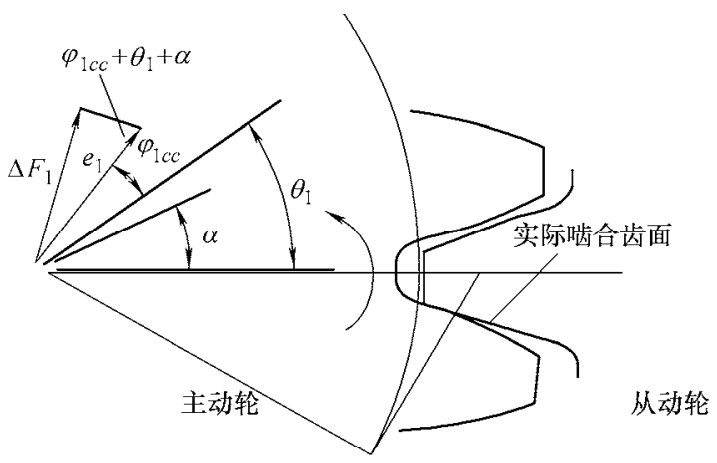

(a) 主动轮逆顺时针方向旋转

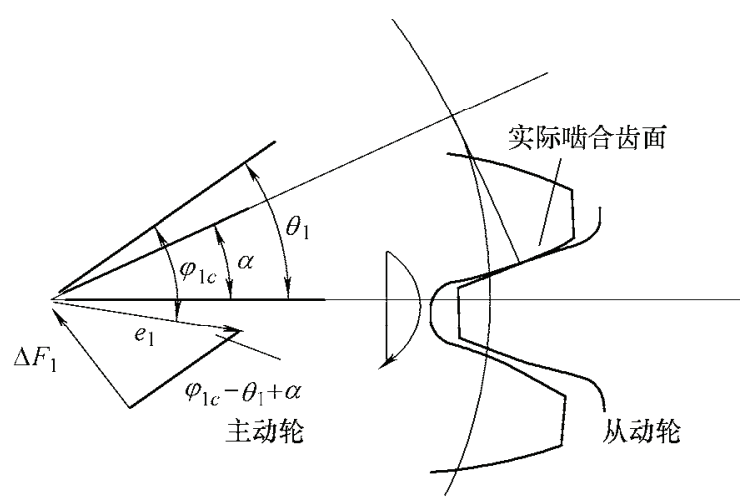

(b) 主动轮顺时针方向旋转

图 3 主动轮偏心误差下啮合线增量计算模型
心误差及其初始相位; $\alpha$ 为齿轮副压力角。如图 3 中 $\Delta F_{1}$ 为主动轮偏心导致的啮合线增量。

同理, 定义从动轮几何中心与其实际旋转中心 的偏移量 $e_{2}$ (图 4)为从动轮综合偏心误差, 则其啮合 线增量计算如下

$$
\left[\begin{array}{l}
\Delta F_{2 n} \\
\Delta F_{2 s} \\
\Delta F_{1 n}^{0} \\
\Delta F_{2 s}^{0}
\end{array}\right]=e_{2}\left[\begin{array}{c}
\sin \left(\varphi_{2 c c}+\theta_{2}-\alpha\right) \\
-\sin \left(\varphi_{2 c}-\theta_{2}-\alpha\right) \\
\sin \left(\theta_{2}-\alpha\right) \\
-\sin \left(-\theta_{2}-\alpha\right)
\end{array}\right]
$$

式中, $\Delta F_{2 n}$ 为从动轮逆时针旋转时偏心导致的啮合 线增量; $\Delta F_{2 s}$ 为从动轮顺时针旋转时偏心导致的啮 合线增量; $\Delta F_{2 n}^{0}$ 为从动轮逆时针旋转初始时刻时偏 心导致的啮合线增量; $\Delta F_{2 s}^{0}$ 为从动轮顺时针旋转初 始时刻时偏心导致的啮合线增量; $\varphi_{2 c c}$ 为主动轮逆 时针旋转时从动轮任意时刻的转动角位移; $\varphi_{2 c}$ 为主 动轮顺时针旋转时从动轮任意时刻转动角位移; $e_{2}$ 和 $\theta_{2}$ 为从动轮偏心误差及其初始相位。图 4 中 $\Delta F_{2}$ 为从动轮偏心导致的啮合线增量。

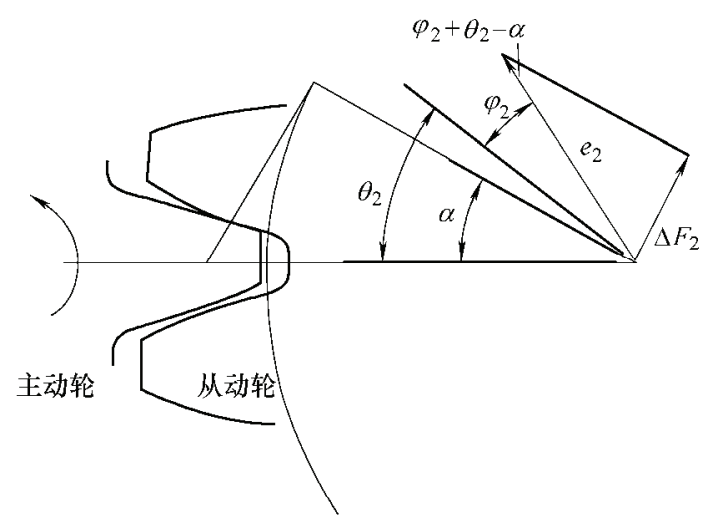

(a) 主动轮逆时针方向旋转

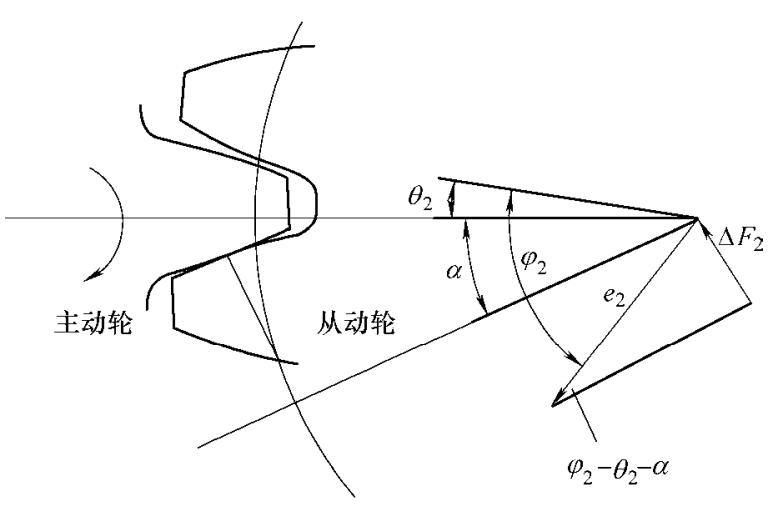

(b) 主动轮顺时针方向旋转

图 4 从动轮偏心误差下啮合线增量计算模型

定义仅由偏心引起的传动误差为无负载传动误 差(后面试验研究的由偏心误差, 刚度, 负载及时变 侧隙引起的传动误差定义为动态传动误差), 将主动 
轮转动方向不同情况下的主从动轮啮合线增量转化 为从动轮旋转角度, 则可分别得到主动轮逆时针与 顺时针方向旋转时的理论无负载传动误差

$$
\begin{aligned}
& \Delta \varphi_{c c}=\frac{180}{\pi R_{2} \cos \alpha}\left(\sum_{i=1}^{2} \Delta F_{i n}-\sum_{i=1}^{2} \Delta F_{i n}^{0}\right) \\
& \Delta \varphi_{c}=\frac{180}{\pi R_{2} \cos \alpha}\left(\sum_{i=1}^{2} \Delta F_{i s}-\sum_{i=1}^{2} \Delta F_{i s}^{0}\right)
\end{aligned}
$$

式中, $\Delta \varphi_{c c}$ 为主动轮逆时针旋转时理论无负载传动 误差; $\Delta \varphi_{c}$ 为主动轮顺时针旋转时理论无负载传动 误差, 单位均为度。

\section{2 侧隙理论计算}

齿轮副总侧隙 ${ }^{[12]}$ 通常包括常值侧隙和周期性 时变侧隙, 其中常值侧隙主要由齿厚偏差、中心距 误差等引起; 时变侧隙主要由轴承、轴及齿轮等制 造、装配偏心误差引起。当主动轮逆时针旋转时, 时变侧隙具体计算如下 ${ }^{[13]}$

$$
\begin{gathered}
j_{v}=\left(\sqrt{b^{2}+c^{2}}-a\right) \times \frac{180 \times 2 \sin \alpha}{\pi R_{2} \cos \alpha} \\
b=a+e_{2} \cos \left(\varphi_{2 c c}+\theta_{2}\right)-e_{1} \cos \left(\varphi_{1 c c}+\theta_{1}\right) \\
c=e_{1} \sin \left(\varphi_{1 c c}+\theta_{1}\right)-e_{2} \sin \left(\varphi_{2 c c}+\theta_{2}\right)
\end{gathered}
$$

将上式用牛顿二项式定理展开, 得

$$
j_{v}=\frac{180 \times 2 \sin \alpha}{\pi R_{2} \cos \alpha} \times
$$

$\left\{e_{2} \cos \left(\varphi_{2 c c}+\theta_{2}\right)-e_{1} \cos \left(\varphi_{1 c c}+\theta_{1}\right)+\right.$

$\frac{1}{2} \frac{e_{1} \sin \left(\varphi_{1 c c}+\theta_{1}\right)-e_{2} \sin \left(\varphi_{2 c c}+\theta_{2}\right)}{\left(a+e_{2} \cos \left(\varphi_{2 c c}+\theta_{2}\right)-e_{1} \cos \left(\varphi_{1 c c}+\theta_{1}\right)\right)}+$

$\left.\frac{1}{2} \frac{\left[e_{1} \sin \left(\varphi_{1 c c}+\theta_{1}\right)-e_{2} \sin \left(\varphi_{2 c c}+\theta_{2}\right)\right]^{2}}{\left[a+e_{2} \cos \left(\varphi_{2 c c}+\theta_{2}\right)-e_{1} \cos \left(\varphi_{1 c c}+\theta_{1}\right)\right]^{3}}+\cdots\right\}$

由于 $e_{1} \ll a, e_{2} \ll a$, 因此

$$
\begin{gathered}
j_{v} \approx \frac{180 \times 2 \sin \alpha}{\pi R_{2} \cos \alpha} \times \\
{\left[e_{2} \cos \left(\varphi_{2 c c}+\theta_{2}\right)-e_{1} \cos \left(\varphi_{1 c c}+\theta_{1}\right)\right]}
\end{gathered}
$$

由式(8)易知初始时变侧隙为

$$
j_{v 0}=\frac{180 \times 2 \sin \alpha}{\pi R_{2} \cos \alpha} \times\left(e_{2} \cos \theta_{2}-e_{1} \cos \theta_{1}\right)
$$

因此, 齿轮副总侧隙及初始总侧隙可表示为

$$
\begin{gathered}
j_{\varphi}=j_{v}+j_{c} \\
j_{\varphi 0}=j_{v 0}+j_{c}
\end{gathered}
$$

式中, $j_{v} 、 j_{v 0} 、 j_{\varphi} 、 j_{\varphi 0}$ 及 $j_{c}$ 分别为齿轮副时变侧 隙、初始时变侧陌、总侧隙、初始总侧隙及常值侧
隙, 单位均为度; $R_{2}$ 为从动轮分度圆半径。

\section{3 双向无负载传动误差与侧隙等效关系}

当主动轮旋转一个大周期时, 可知

$$
\left[\begin{array}{l}
\varphi_{1 c c} \\
\varphi_{2 c c}
\end{array}\right]=\left[\begin{array}{l}
2 \pi n-\varphi_{1 c} \\
\frac{2 \pi n}{i}-\varphi_{2 c}
\end{array}\right]
$$

式中, $n$ 代表齿轮副旋转一个大周期主动轮需要转 动的圈数, $i$ 为齿轮副传动比。

通过上述计算公式可知

$$
\begin{gathered}
\Delta \varphi_{c c}+j_{\varphi}=\Delta \varphi_{c c}+j_{v}+j_{c}= \\
\frac{180}{\pi R_{2} \cos \alpha} \times\left[e_{1} \sin \left(\varphi_{1 c c}+\theta_{1}+\alpha\right)+\right. \\
e_{2} \sin \left(\varphi_{2 c c}+\theta_{2}-\alpha\right)-e_{1} \sin \left(\theta_{1}+\alpha\right)- \\
\left.e_{2} \sin \left(\theta_{2}-\alpha\right)\right]+\frac{180 \times 2 \sin \alpha}{\pi R_{2} \cos \alpha} \times \\
{\left[e_{2} \cos \left(\varphi_{2 c c}+\theta_{2}\right)-e_{1} \cos \left(\varphi_{1 c c}+\theta_{1}\right)\right]+j_{c}=} \\
\frac{180}{\pi R_{2} \cos \alpha} \times\left[-e_{1} \sin \left(\varphi_{1 c}-\theta_{1}+\alpha\right)-\right. \\
e_{2} \sin \left(\varphi_{2}-\theta_{2}-\alpha\right)+e_{1} \sin \left(-\theta_{1}+\alpha\right)+ \\
\left.e_{2} \sin \left(-\theta_{2}-\alpha\right)\right]+\frac{180 \times 2 \sin \alpha}{\pi R_{2} \cos \alpha} \times \\
\left(e_{2} \cos \theta_{2}-e_{1} \cos \theta_{1}\right)+j_{c}=\Delta \varphi_{c}+j_{\varphi 0}
\end{gathered}
$$

因此

$$
j_{\varphi}=\left(\Delta \varphi_{c}+j_{\varphi 0}\right)-\Delta \varphi_{c c}
$$

综上, 具有偏心误差的齿轮副总侧隙可通过双 向无负载传动误差(即逆时针传动误差及含初始侧 隙的顺时针传动误差)得到。因此, 通过正反向传动 误差的测试, 可直接得到连续的侧隙曲线, 据此可 确定变齿厚齿轮轴向移动量。

\section{3 双向传动误差及侧隙测量}

\section{1 试验装置及参数}

消隙试验装置 ${ }^{[14-15]}$ 如图 5 所示, 试验系统包 括驱动电动机及其控制器 1 、新型变齿厚齿轮副、 绝对式编码器 1 和 2 、负载电动机及其控制器 2 、 滚珠花键副、数据采集系统、直线执行器、消隙 控制器以及上位机等。通过控制器 1 控制驱动电 动机旋转, 控制器 2 控制负载电动机提供试验所 需负载, 编码器 1 和 2 分别测量主从动轮转动角 度, 从而获得试验传动误差曲线。此外, 本装置 中的变齿厚齿轮副属于渐开线齿轮副的一种, 因 此上述建立的传动误差及侧隙理论模型仍然适 用。双向传动误差及侧隙的测试工况及齿轮副参 数如表 1 所示。 

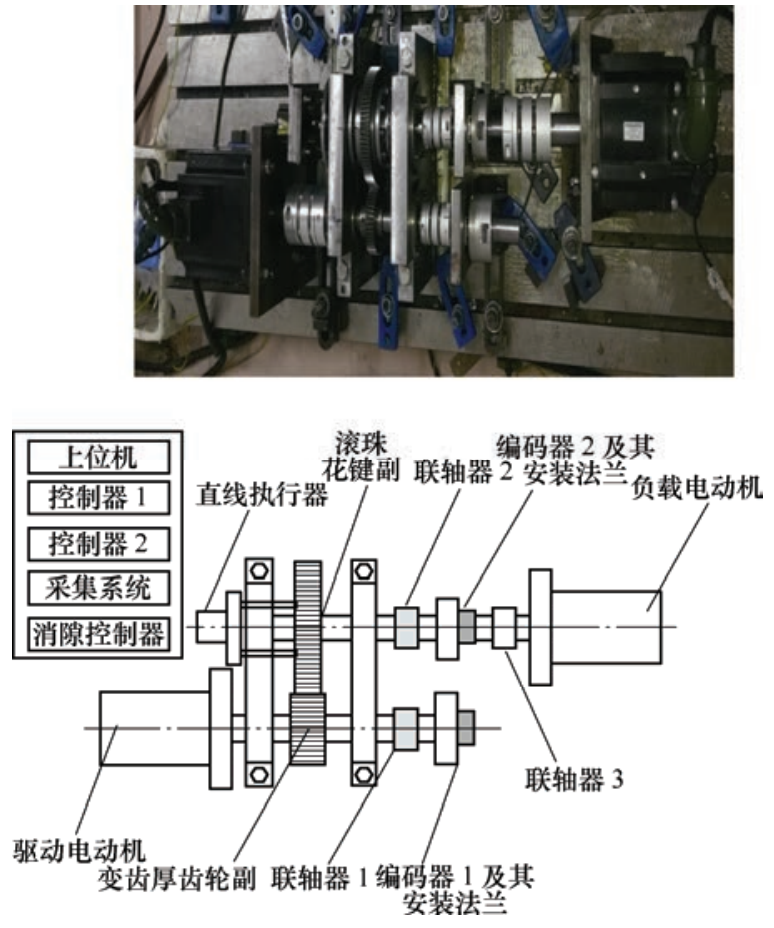

图 5 消隙试验装置图

表 1 试验工况及齿轮副基本参数

\begin{tabular}{lc}
\hline \multicolumn{1}{c}{ 参数 } & 数值 \\
\hline 主动轮齿数 & 45 \\
从动轮齿数 & 80 \\
模数 $/ \mathrm{mm}$ & 2 \\
压力角 $/\left({ }^{\circ}\right)$ & 20 \\
齿宽 $/ \mathrm{mm}$ & 20 \\
左右螺旋角 $/\left({ }^{\circ}\right)$ & 3 \\
齿顶圆直径 $/ \mathrm{mm}$ & 94 \\
齿根圆直径 $/ \mathrm{mm}$ & 85 \\
输入转速 $/(\mathrm{r} / \mathrm{min})$ & 10 \\
负载力矩 $/(\mathrm{N} \cdot \mathrm{m})$ & 0 \\
\hline
\end{tabular}

\section{2 双向传动误差及侧隙测量}

首先在主动轮逆时针方向齿面贴合下，主动轮逆 时针方向无负载力矩低速旋转一个大周期(根据表 1 齿 轮副参数可知, 主动轮旋转 16 圈为一个大周期)得到齿 轮副逆时针传动误差曲线, 主动轮再顺时针方向无负 载力矩低速旋转一个大周期得到包含初始侧隙的顺时 针传动误差曲线, 如图 6 所示。并定义在无负载力矩 工况下的传动误差曲线为无负载传动误差曲线。

根据推导的传动误差和偏心误差关系, 将 式 (4)、(5)及双向无负载传动误差曲线导入 Matlab 拟合工具箱, 可拟合得到齿轮副偏心误差及初始相 位, 拟合度均在 95\%以上(具体拟合方法见文献[11]), 然后通过式(8)、(9), 可得到齿轮副的时变侧隙和初始 时变侧隙。再通过顺时针传动误差曲线中读取的初始 侧隙、初始时变侧隙和式(11), 得到常值侧隙值, 最 终根据式(10)获得齿轮副侧隙曲线, 并定义此处得到
的侧隙为拟合法侧隙曲线, 如图 6 所示。

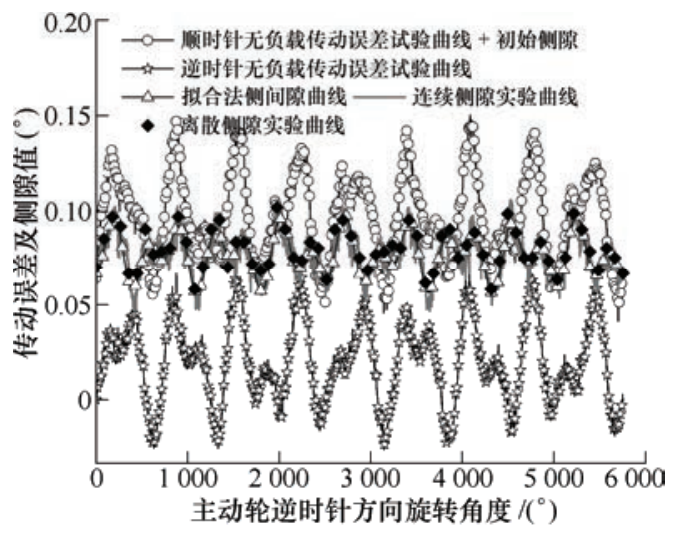

图 6 双向传动误差及侧隙曲线

通过双向无负载传动误差试验曲线及式(14)得 到连续侧隙试验曲线。此外, 试验采用了机械滞后 回差法 ${ }^{[16]}$ 获得齿轮副离散的侧隙曲线。结果表明, 三种方法所得到的侧隙曲线具有良好的一致性。

在得到齿轮副连续侧隙曲线后, 因侧隙控制程 序中须先设置初始相位, 以便与连续的侧隙曲线进 行对应。由于绝对式编码器提供当前主从动轮绝对 位置值, 因此通过与上述拟合得到的初始相位叠加, 即得到每次重新启动后的初始相位, 从而确定齿轮 副各个角位置与连续侧隙曲线的对应关系, 而不需 要每次启动后重新回到参考点。

\section{3 消隙执行器轴向移动位移}

根据式(1), 由图 6 所示的连续侧隙试验曲线对 应的电控消隙机构轴向位移如图 7a 所示。图 7b 为 轴向移动位移的快速傅里叶变换图, 由图知, 轴向 移动位移频率与表 1 中主从动轮转速相对应的转频 及其相应的倍频一致。由于消隙执行器通常在传动 末级执行消隙, 其转速通常不高(即大周期频率较 低), 因此轴向移动执行器易满足消隙响应性要求。 若需要在速度、载荷更高的工况下消隙时, 则需要 相应提高消隙执行器速度和负载能力。

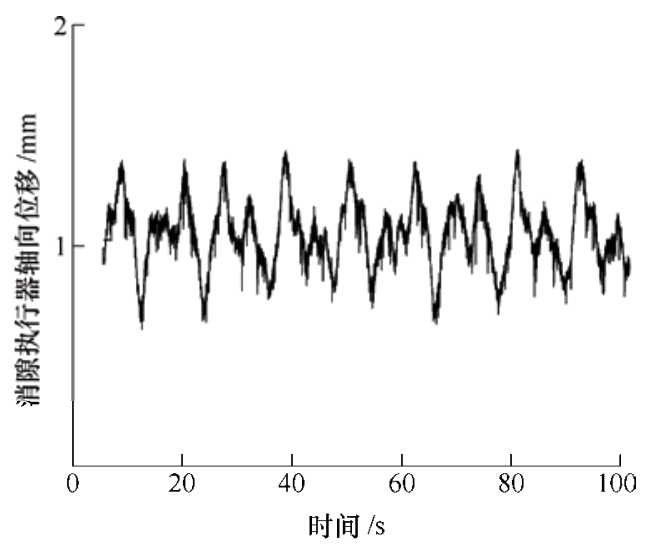

(a) 时域内轴向位移 


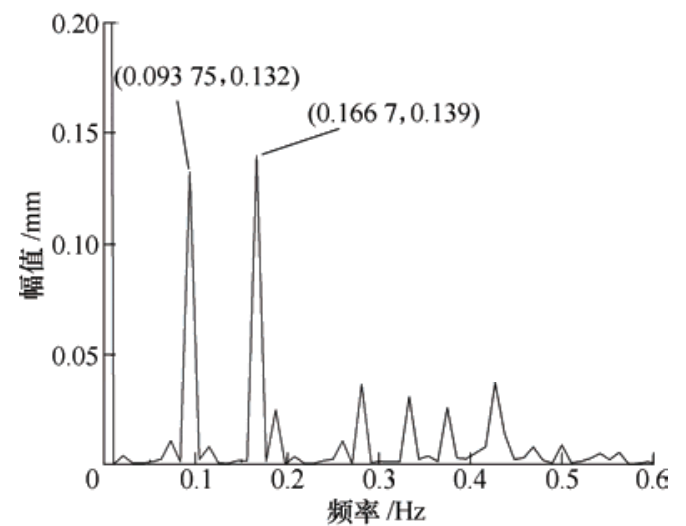

(b) 频率内轴向位移幅值

图 7 消隙机构轴向移动位移及快速傅里叶变换

\section{4 交变负载下无消隙控制的动态 传动误差试验分析}

\section{1 未消隙控制下动态传动误差试验曲线}

如图 8 所示, 试验分别测得了主动轮逆时针方 向旋转时, $\pm 5 \mathrm{~N} \cdot \mathrm{m}$ 常值负载及 $5 \mathrm{~N} \cdot \mathrm{m}$ 交变负 载下的动态传动误差曲线。此处定义负载方向与从 动轮转动方向相反时为正, 负载方向与从动轮转动 方向相同时为负。其中正向和反向负载下的动态传 动误差波形分别与图 6 所示的无负载下逆时针和顺 时针传动误差波形一致, 但分别向上和向下偏移约 $0.03^{\circ}$, 偏移量主要由两编码器间零件(输出轴、膜片 联轴器、滚珠花键副、齿轮副等)刚度和负载引起。 而交变负载动态传动误差在正向和反向负载下的动 态传动误差之间, 且当负载小于零附近位置时, 动 态传动误差出现明显突变, 说明此时因负载方向变 化而导致轮齿脱啮, 形成相反齿面啮合, 此时由于 齿轮副存在侧隙造成输出轴输出位移不连续, 在负 载反向时动态传动误差产生突变, 突变量即为该角 位移位置处的侧隙值, 如图 8 所示。

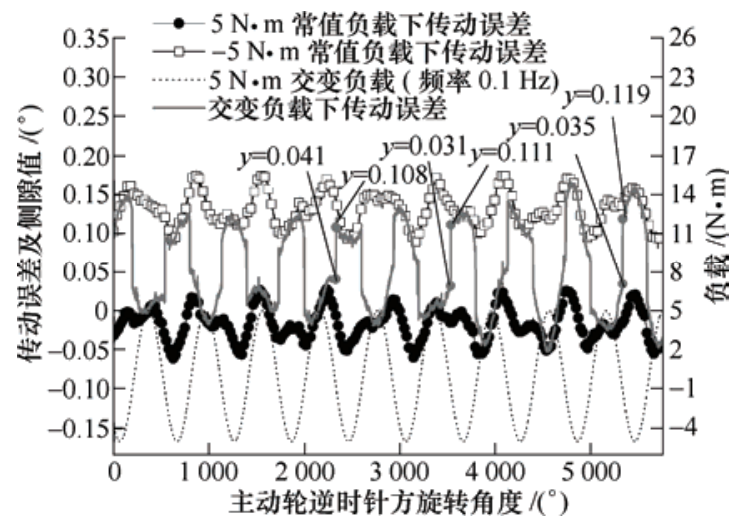

图 $85 \mathrm{~N} \cdot \mathrm{m}$ 交变负载及动态传动误差

\section{2 负载幅值的影响}

如图 9、10 所示, 在上述试验基础上, 分别进 行了 $1.5 \mathrm{~N} \cdot \mathrm{m}$ 以及 $0.4 \mathrm{~N} \cdot \mathrm{m}$ 不同负载幅值的正 向、反向及交变负载下的动态传动误差。可以看出 随着负载幅值变小, 齿轮副、轴、滚珠花键副及联 轴器等零件变形减小, 交变负载下的动态传动误差 峰-峰值随之减小。此外, 从图 9 中的交变负载动态 传动误差可知，反向负载的啮合区间明显小于正向 负载的啮合区间, 在反向负载为 $-0.5 \mathrm{~N} \cdot \mathrm{m}$ 时, 动 态传动误差才出现跳变, 表明反向负载下齿背啮合 需克服一定摩擦阻尼。

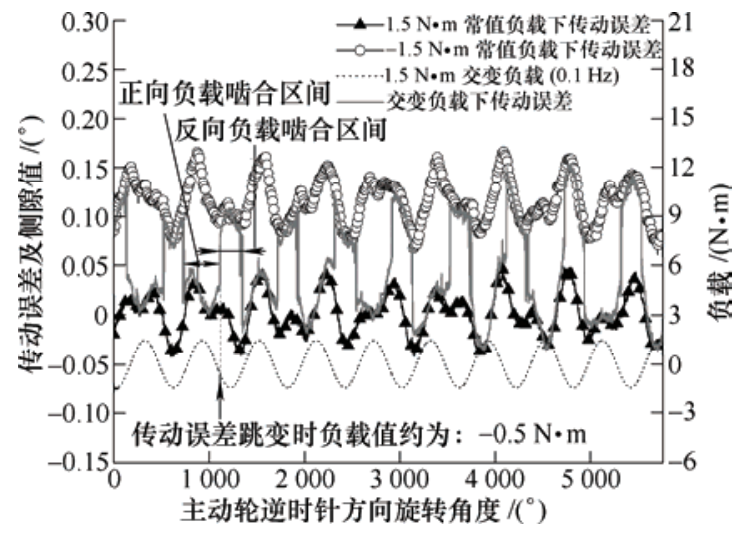

图 $91.5 \mathrm{~N} \cdot \mathrm{m}$ 交变负载及动态传动误差

当交变负载或负常值负载幅值为 $0.4 \mathrm{~N} \cdot \mathrm{m}$ 时, 如图 10 所示, 此时反向负载幅值小于 $0.5 \mathrm{~N} \cdot \mathrm{m}$, 不足以克服摩擦阻尼, 因此不能形成如图 8 与图 9 中的稳定的齿背啮合, 而此时动态传动误差出现明 显的波动, 波动最大峰值达到顺时针无负载传动误 差值, 表明齿轮在传动过程中出现脱啮、单边冲击 和双边冲击。

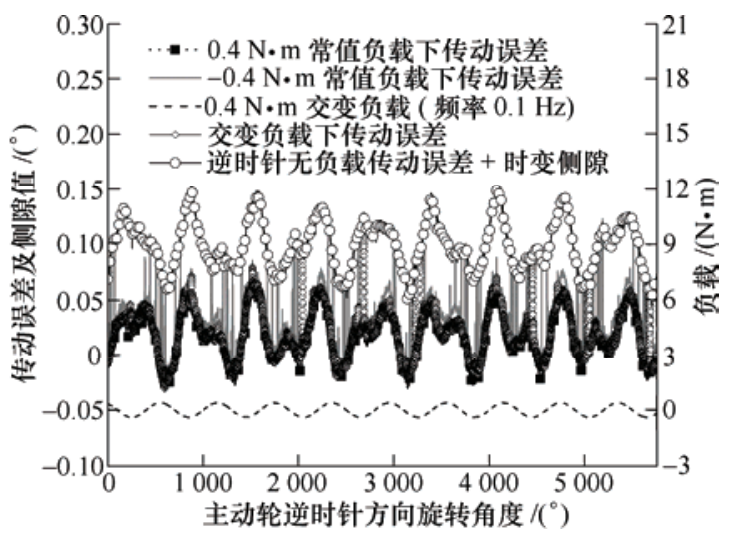

图 $100.4 \mathrm{~N} \cdot \mathrm{m}$ 交变负载及动态传动误差

\section{3 负载频率的影响}

如图 11 所示, 当交变负载幅值大于 $0.5 \mathrm{~N} \cdot \mathrm{m}$ 且交变负载变化频率为 $1 \mathrm{~Hz}$ 时(相对于从动轮转频 较大时), 动态传动误差上沿与相应幅值的负常值负 
载时的动态传动误差吻合, 下沿与相应幅值正的常 值负载时的动态传动误差吻合。当交变负载幅值小 于 $0.5 \mathrm{~N} \cdot \mathrm{m}$ 时, 如图 12 所示, 其上沿出现明显波 动, 表明齿轮在传动过程中出现脱啮、单边和双边 冲击, 不能形成稳定的齿背啮合。

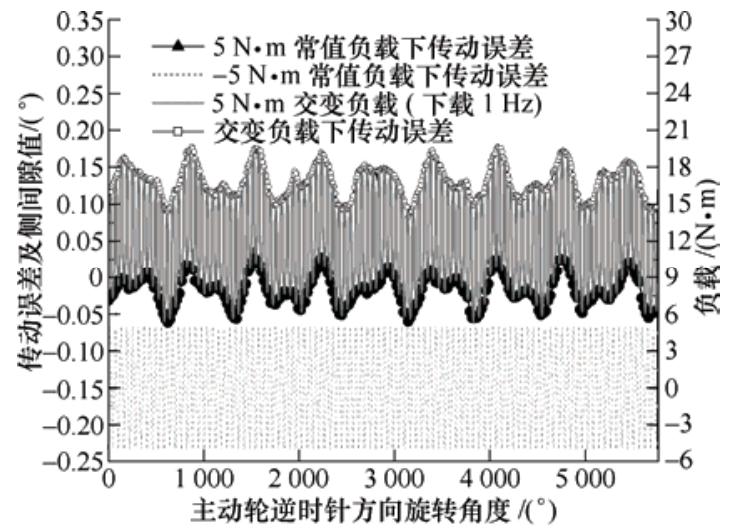

图 $115 \mathrm{~N} \cdot \mathrm{m}$ 交变负载及动态传动误差

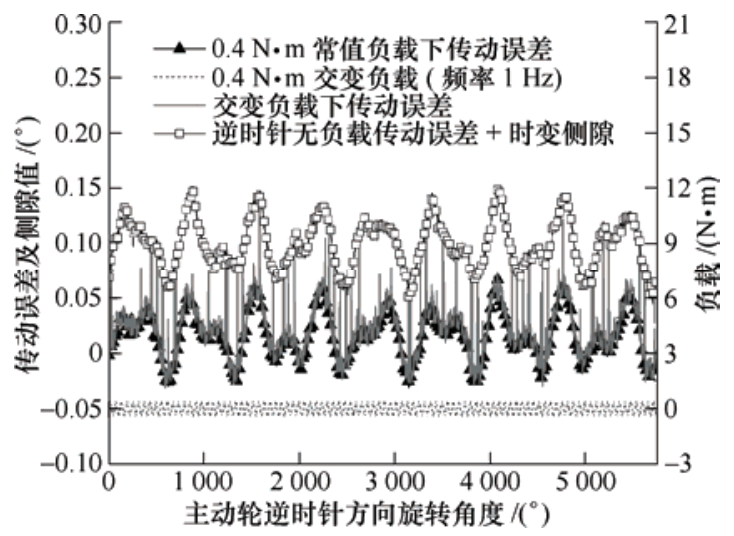

图 $120.4 \mathrm{~N} \cdot \mathrm{m}$ 交变负载下的动态传动误差

综上可知, 交变负载下侧隙对传动系统动态传 动误差有明显影响, 有必要对侧隙进行控制。

5 交变负载下的消隙控制与动态 传动误差试验分析

\section{1 消隙控制下的动态传动误差试验曲线}

\subsection{1 $0.1 \mathrm{~Hz}$ 交变负载动态传动误差}

如图 13、14 分别所示为幅值 $5 \mathrm{~N} \cdot \mathrm{m}$ 和 $1.5 \mathrm{~N} \cdot \mathrm{m}$ 的交变负载(频率 $0.1 \mathrm{~Hz}$ )下基于主动轮角 位移消隙控制的动态传动误差曲线。对比图 8 及图 9 未消隙的动态传动误差曲线可知, 消隙后动态传动 误差曲线峰-峰值明显减小, 传动精度得到提高。同 时在负载反向时, 没有明显出现图 8 和图 9 中类似 的因侧隙导致的跳变, 因此说明了通过上述消隙策 略的控制后侧隙得到有效控制。此外, 图 15 为 $0.4 \mathrm{~N} \cdot \mathrm{m}$ 交变负载(频率 $0.1 \mathrm{~Hz}$ )下的消隙控制动态
传动误差曲线, 对比图 10 可知, 因侧隙导致的波动 基本消失，同样说明了侧隙得到很好地控制。

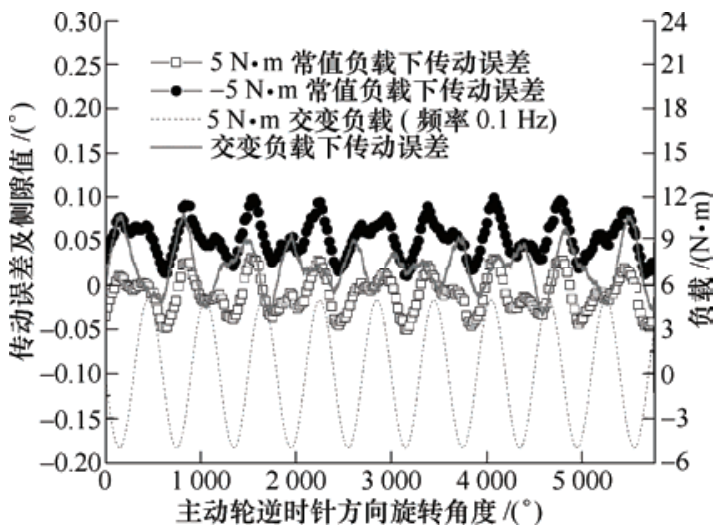

图 $135 \mathrm{~N} \cdot \mathrm{m}$ 交变负载下消隙控制的动态传动误差

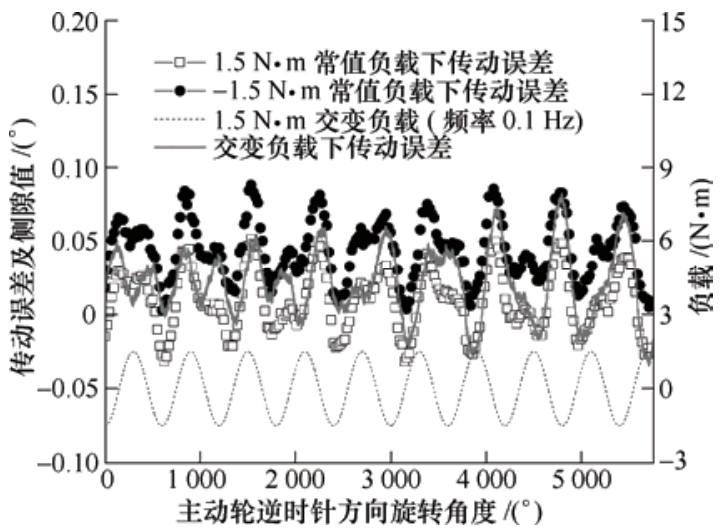

图 $141.5 \mathrm{~N} \cdot \mathrm{m}$ 交变负载下消隙控制的动态传动误差

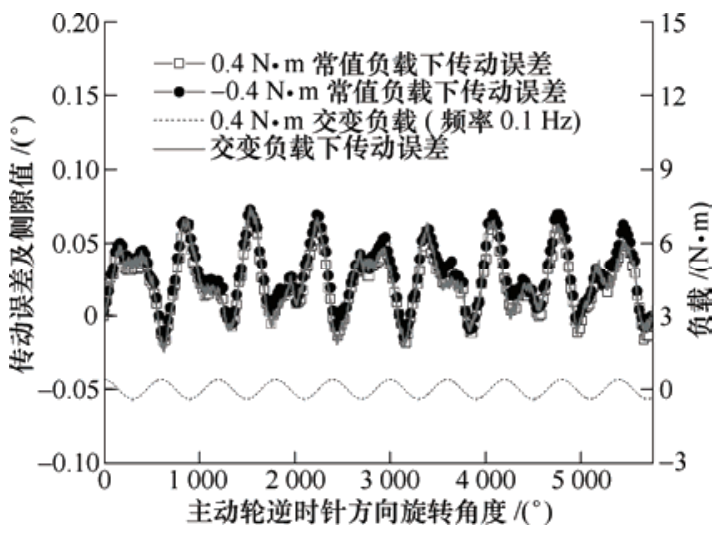

图 $150.4 \mathrm{~N} \cdot \mathrm{m}$ 交变负载下消隙控制的动态传动误差

\subsection{2 $1 \mathrm{~Hz}$ 交变负载动态传动误差}

如图 16 所示，当交变负载幅值为 $5 \mathrm{~N} \cdot \mathrm{m}$ 且交 变负载频率为 $1 \mathrm{~Hz}$ 时(负载频率相对于从动轮转频 较大时), 对比图 11 可知, 其动态传动误差上沿同 样与相应负常值负载动态传动误差吻合，下沿与相 应正的常值负载动态传动误差曲线吻合, 但动态传 动误差峰-峰值明显减小, 且上下沿动态传动误差波 形一致，说明周期性时变侧隙基本消除，而导致动 态传动误差波动主要原因是负载力矩和系统刚度引 
起的变形随负载大小变化所致。当负载为 $0.4 \mathrm{~N} \cdot \mathrm{m}$ 时, 由于负载引起的变形很小, 因此此时动态传动 误差变化区域随之减小, 如图 17 所示。同时对比 图 12 可知, 图 17 中动态传动误差波动现象基本消 失, 因此图 16 和图 17 两种情况均证明通过消隙后 齿轮副侧隙得到有效控制, 系统传动精度得到改善。

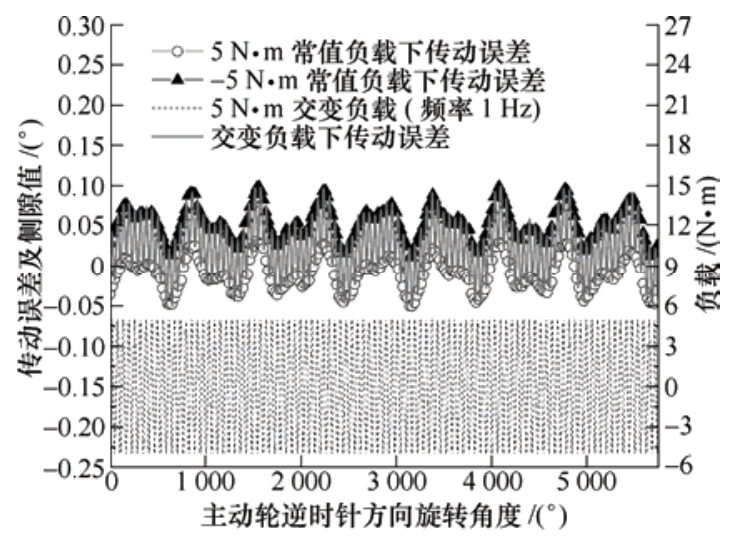

图 $165 \mathrm{~N} \cdot \mathrm{m}$ 交变负载下消隙控制的动态传动误差

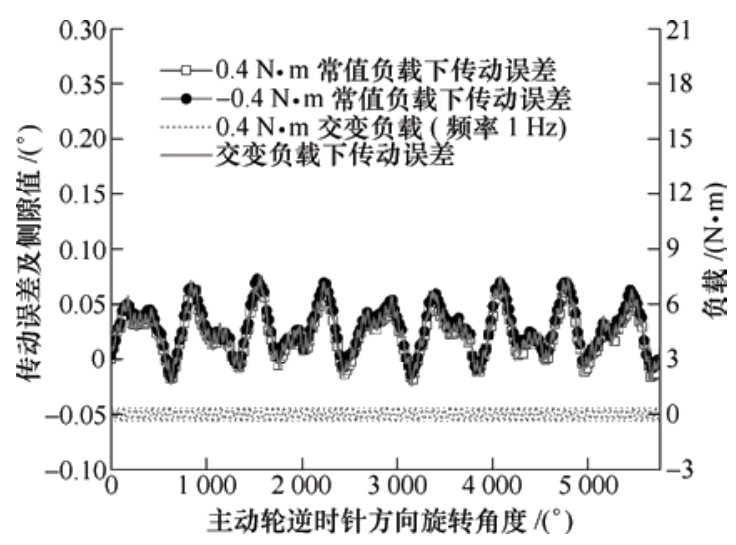

图 $170.4 \mathrm{~N} \cdot \mathrm{m}$ 交变负载下消隙控制的动态传动误差

\section{2 消隙前后交变负载动态传动误差对比}

根据上述所有测试的动态传动误差曲线, 对其 均方根值, 均值及峰-峰值进行对比, 如表 2 所示。

表 2 消隙前后动态传动误差对比

\begin{tabular}{ccccc}
\hline \multirow{2}{*}{ 交变负载 } & & \multicolumn{3}{c}{ 动态传动误差 $/\left(^{\circ}\right)$} \\
\cline { 3 - 5 } & & 均方根值 & 均值 & 峰-峰值 \\
\hline \multirow{2}{*}{$5 \mathrm{~N} \cdot \mathrm{m}, 0.1 \mathrm{~Hz}$} & 消隙 & 0.0324 & 0.0201 & 0.1161 \\
& 未消隙 & 0.0795 & 0.0529 & 0.2187 \\
$5 \mathrm{~N} \cdot \mathrm{m}, 1 \mathrm{~Hz}$ & 消隙 & 0.0399 & 0.0235 & 0.1528 \\
& 未消隙 & 0.0818 & 0.0505 & 0.2337 \\
$1.5 \mathrm{~N} ・ \mathrm{~m}, 0.1 \mathrm{~Hz}$ & 消隙 & 0.0320 & 0.0220 & 0.1173 \\
& 未消隙 & 0.0658 & 0.0448 & 0.1905 \\
$0.4 \mathrm{~N} \cdot \mathrm{m}, 0.1 \mathrm{~Hz}$ & 消隙 & 0.0320 & 0.0238 & 0.1001 \\
& 未消隙 & 0.0295 & 0.0200 & 0.1412 \\
$0.4 \mathrm{~N} \cdot \mathrm{m}, 1 \mathrm{~Hz}$ & 消隙 & 0.0332 & 0.0259 & 0.1006 \\
& 未消隙 & 0.0339 & 0.0231 & 0.1720 \\
\hline
\end{tabular}

易知, 消隙前齿轮副动态传动误差均方根值及均值 均随着负载增大而增大, 消隙后动态传动误差均方 根值和均值均比较稳定, 分别为 $0.032^{\circ}$ 和 $0.022^{\circ}$ 左 右, 而由于消隙后动态传动误差的跳变或波动基本 消除, 因此可知由侧隙部分引起的传动误差得到了 较好控制, 传动精度均有所提高, 证明了电控消隙 机构的有效性。由于本文旨在实现和验证电控消隙 机构的消隙功能, 齿轮等零件的加工和装配精度采 用普通精度(齿轮精度为 7 级), 因此, 由于偏心等 引起的大周期传动误差较大, 但消隙效果明显。

\section{6 结论}

(1) 提出一种基于变齿厚齿轮侧隙控制方法: 在得到变齿厚齿轮副连续的侧隙曲线后，根据实时 检测到的主动轮角位移, 调整变齿厚齿轮主从动齿 轮的相对轴向位置关系, 实现齿轮副啮合侧隙的 控制。

(2) 推导具有双偏心误差齿轮副的双向无负载 传动误差及侧隙理论计算公式及其等效关系, 并通 过测得的双向传动误差试验曲线得到连续侧隙曲 线, 相比机械滞后回差法测得的离散试验侧隙曲线, 该方法效率更高, 并能获得更全面的齿轮副啮合侧 隙数据。

（3）在交变负载条件下进行了侧隙控制试验, 结果表明: 由于控制了侧隙, 在齿轮副脱啮时动态 传动误差出现的跳变或波动现象得到良好改善, 动 态传动误差峰-峰值减小, 系统动态传动精度得到提 高, 表明该方法能有效控制侧隙。

（4）当交变负载大于 $0.5 \mathrm{~N} \cdot \mathrm{m}$, 且频率为 $1 \mathrm{~Hz}$ 时 (即相对于从动轮转频较大时), 其动态传动误差 上沿与相应负常值负载下的动态传动误差吻合, 下 沿与相应正的常值负载下的动态传动误差吻合。

(5) 在交变负载大于 $0.5 \mathrm{~N} \cdot \mathrm{m}$ 工况下, 未消隙 控制的齿轮副动态传动误差在反向负载时出现明显 跳变, 其跳变量为该位置的侧隙值; 当负载幅值小 于 $0.5 \mathrm{~N} \cdot \mathrm{m}$ 时, 负载力矩不足以克服摩擦阻尼, 反向负载下不能形成稳定的齿背啮合, 因此此时传 动误差未出现跳变, 但由于侧隙的原因, 传动误差 出现波动现象(拍击), 其波动最大峰值与侧隙值一 致, 说明齿轮在传动过程中出现脱啮、单边冲击和 双边冲击。

\section{参 考 文 献}

[1] WANG Guangjian, CHEN Lin, YU Li, et al. Research on 
the dynamic transmission error of a spur gear pair with eccentricities by finite element method[J]. Mechanism and Machine Theory, 2017, 109: 1-13.

[2] 崔亚辉, 刘占生, 叶建槐, 等. 内外激励作用下含侧隙 的齿轮传动系统的分岔和混沌 [J]. 机械工程学报, 2010, 46(11): 129-136.

CUI Yahui, LIU Zhansheng, YE Jianhuai, et al. Bifurcation and chaos of gear transmission system with clearance subjected to internal and external excitation[J]. Journal of Mechanical Engineering, 2010，46(11): 129-136.

[3] 余驰. 机械精密传动消隙结构分析与研究 [J]. 机械研 究与应用, 2016, 1(29): 8-11.

YU Chi. Analysis and study on eliminating clearance structure of mechanical precision transmission[J]. Mechanical Research and Application, 2016, 1(29): 8-11.

[4] MICHAELIC G W. Precision gearing: Theory and practice[M]. New York: John Wiley \& Sons, 1996.

[5] SHI Zhiguang, ZUO Zongyu. Backstepping control for gear transmission servo systems with backlash nonlinearity[J]. IEEE Transaction on Automation Science and Engineering, 2015, 12(2): 752-757.

[6] MARTIN W, MUSA J. Backlash compensation in gear trains by means of open-loop modification of the input trajectory[J]. Journal of Mechanical Design, 2003, 125(3): 115-120.

[7] SATO K, MURAYAMA Y, IMADA S, et al. Control and elimination of lead screw backlash for ultra-precision positioning[J]. JSME International Journal，1995，38(1): 36-41.

[8] 张珍珍, 彭超, 邵仕泉. 基于转速差调节和模型预测控 制的双电动机伺服系统消隙控制 [J]. 微电动机, 2017, 50(1): 54-58.

ZHANG Zhenzhen, PENG Chao, SHAO Shiquan. A rotation speed offset regulation and model predictive control based anti-backlash control approach for dual motor servo system[J]. Macromotors, 2017, 50(1): 54-58.

[9] 陈威, 吴益飞, 杜仁慧, 等. 双电动机同步驱动伺服系 统故障诊断与容错控制 [J]. 控制理论与应用, 2014, 31(1): 27-34.

CHEN Wei, WU Yifei, DU Renhui, et al. Fault diagnosis and fault tolerant control for the servo system driven by two motors synchronously[J]. Control Theory and Appllication, 2014, 31(1): 27-34.

[10] YU Li, WANG Guangjian, ZOU Shuaidong. The calculation of meshing efficiency of a new type of conical involute gear[J]. Strojniski Vestnik-Journal of Mechanical Engineering, 2017, 63(5): 320-330.

[11] WANG Guangjian, YU Li, ZOU Shuaidong. The experimental research on gear eccentricity error of backlash-compensation gear device based on transmission error[J]. International Journal of Precision Engineering and Manufacturing, 2018, 19(1): 5-12.

[12] GEORGE W M. Correlation of probabilistic backlash with measurements[J]. Mechanism and Machine Theory, 1973, 8: 161-173.

[13] 吴慈生. 齿轮偏心误差所引起的传动误差 $[\mathrm{J}]$. 南京工 学院学报, 1982(4): 133-145.

WU Cisheng. Research on transmission error by eccentric gear[J]. Journal of Nanjing Institute of Technology, 1982(4): 133-145.

[14] 王光建. 电控消隙的变齿厚齿轮副: 中国, ZL201210384456.3[P]. 2015-07-15.

WANG Guangjian. Variable tooth thickness gear pair for anti-backlash based on eclectric control : China , ZL201210384456.3[P]. 2015-07-15.

[15] YU Li, WANG Guangjian, HE Liangliang. The electrically controlling anti-backlash based on variable tooth thickness gear[C]// The International Conference on Gears, December 5-7, 2015, TUM, Munich. Dusseldorf: VDI, 2015: 591-600.

[16] 齿轮手册编委会. 齿轮手册 [M]. 北京: 机械工业出版 社, 2006.

Gear Manual Editorial Board. Gear manual[M]. Beijing: China Machine Press, 2006.

作者简介: 邹帅东, 男, 1987 年出生, 博士研究生。主要研究方向为精 密传动与控制。

E-mail: 20150701001@cqu.edu.cn

王光建(通信作者), 男, 1973 年出生, 博士, 教授, 博士研究生导师。 主要研究方向为精密传动与控制。

E-mail: gjwang@cqu.edu.cn

蒋宇将, 男, 1994 年出生, 博士。主要研究方向为精密传动与控制。

E-mail:772071997@qq.com 